\title{
À se tordre de rire : la représentation du corps dans les films comiques américains et français
}

\author{
Lauretta Clough et Caroline Eades
}

\section{OpenEdition}

\section{Journals}

Édition électronique

URL : http://journals.openedition.org/recherchestravaux/295

DOI : 10.4000/recherchestravaux.295

ISSN : 1969-6434

Éditeur

UGA Éditions/Université Grenoble Alpes

\section{Édition imprimée}

Date de publication : 30 mai 2005

Pagination : 273-289

ISBN : 0151-1874

ISSN : 0151-1874

Référence électronique

Lauretta Clough et Caroline Eades, «À se tordre de rire : la représentation du corps dans les films comiques américains et français », Recherches \& Travaux [En ligne], 67 | 2005, mis en ligne le 30 septembre 2008, consulté le 08 septembre 2020. URL : http://journals.openedition.org/ recherchestravaux/295; DOI : https://doi.org/10.4000/recherchestravaux.295 


\section{À se tordre de rire: la représentation du corps dans les films comiques américains et français}

Ce que j'en dis n'est qu'entre nous, et j'aurais souhaité de pouvoir un peu vous tirer de l'erreur où vous êtes, et, pour vous divertir, vous mener voir, sur ce chapitre, quelqu'une des comédies de Molière.

MOLIÈRE, Le Malade imaginaire, acte III, sc. 3.

Le cinéma, dans sa constante tension entre représentation réaliste et réagencement du monde perçu, vécu et imaginé, joue-t-il à défier la proposition d'Aristote devenue axiome selon laquelle le rire est le propre de l'homme? De fait, prenant la suite de la tradition établie par la comédie théâtrale et la fable, d'Aristophane à Shakespeare, d'Ésope à La Fontaine, tout comme par les contes de fées et les illustrateurs de presse, les films ont à leur tour permis aux animaux de partager cette spécificité humaine. Ils ont aussi participé à une exploration inverse de la définition du rire, en mettant l'accent non pas tant sur un trait défini comme commun à tous les êtres humains que sur la nature humaine telle qu'elle est définie par ce trait, rejoignant Bergson dans la description de la fonction sociale du rire. À la perspective anthropologique et à l'investigation sociologique sur le rire, s'ajoute la réflexion sur sa fonction esthétique: depuis l'Antiquité, le rire suscite la création artistique par sa double dimension, universelle et particulière, propre à un individu et représentative d'une communauté, proche de l'expérience affective et facteur de distanciation par le biais de l'absurde, l'anthropomorphisme, la caricature, l'inversion carnavalesque, le travestissement ludique ${ }^{\mathrm{I}}$.

I. On s'opposera donc à la définition de G. Mast selon laquelle « un film (ou gag, ou répartie, ou personnage) n'est vraiment amusant que lorsque le public n'est pas conscient qu'il a l'intention d'amuser. [...] Peut-être le seul terme pour décrire le mariage réussi entre intention 
Le cinéma n'a donc pas échappé à la question que pose l'analyse de la comédie au regard des particularismes nationaux ou culturels, en fonction du contexte de création, de production et de réception des films:

[...] dans quelle mesure les nations ou régions individuelles ont-elles leurs propres types de comédie spécifiques? Si ce pour quoi nous rions est une façon de nous définir, par la référence à des caractéristiques propres à un groupe ou extérieures, peut-on identifier des traits comiques particuliers à une nation ou à une autre? (King, p. 157)

La tentative de déterminer des spécificités géoculturelles ne doit pas non plus faire abstraction des évolutions historiques d'un genre dans un contexte particulier: ainsi, pour prendre l'exemple du cinéma français, André Bazin constatait dans les années 1950 que Raimu, Fernandel, Tati et les frères Prévert constituaient une rare exception à la triste postérité de l'école burlesque 3 , alors qu'aujourd'hui certains critiques donnent au film comique une place essentielle dans le cinéma français 4 face à la déferlante des importations américaines 5 .

comique et exécution est l'un des concepts-clés de la Renaissance - sprezzatura. Sprezzatura peut être défini comme l'art qui cache l'art" (A film (or gag, or line, or character) is truly funny when the audience is not conscious that it intends to be funny. [...] Perhaps the only term for describing the successful marriage between comic intention and execution is one of the key concepts of the Renaissance-sprezzatura. Sprezzatura might be defined as the art that conceals art» (The Comic Mind: Comedy and the Movies, Chicago, Chicago University Press, 1997, p. 26).

2. To what extent do individual nations or regions have their own distinct brands of comedy? If what we laugh at is one way of defining us, through reference to in-group or external characteristics, can we identify comic traits particular to one nation or another?

3. «Le burlesque français, si l'on excepte les derniers films de Max Linder réalisés à Hollywood, n’a pratiquement pas dépassé les années 19I4, submergé ensuite par le succès écrasant et justifié - du comique américain. Depuis le parlant, en dehors même de Chaplin, Hollywood est demeuré le maître du cinéma comique» (A. Bazin , "Monsieur Hulot et le temps", Qu'est-ce que le cinéma, Paris, Cerf, rééd. 1994, p. 4I).

4. Voir P. Powrie, French Cinema in the I980s: nostalgia and the crisis of masculinity, Oxford et New York, Clarendon Press, 1997, p. I4I : «Il n’est pas exagéré de dire que les films comiques sont le genre français le plus populaire" (It's no exaggeration to say that comic films are the most popular French genre).

5. R. Prédal, Le Jeune cinéma français, Paris Nathan,/VUEF, 2002: «si le public s’émotionne et s'extasie américain, les Français aiment rire français. Dans les dix premiers films du hit parade ne figurent en effet que deux productions nationales, et ceux-ci sont deux films comiques: 2. La Grande Vadrouille (Gérard Oury, 1966), 9. Les Visiteurs (Jean-Marie Poiré, 1992). Les résultats des dernières années confirment ce choix: en 1998, derrière le "phénomène" Titanic (plus de 20 millions d'entrées!), on avait déjà un beau tir groupé avec 2. Le Dîner de cons (Francis Veber), 3. Les Couloirs du temps, alias Les Visiteurs 2 (Jean-Marie Poiré), suivis en 4. par Taxi (Gérard Pirès). En 1999, le premier au Box Office est Astérix et Obélix contre César (Claude Zidi) et, en 2000, Taxi 2 (Gérard Krawczyk) avec plus de Io millions de spectateurs devant cinq films américains ». 
Deux nuances doivent être aussitôt apportées à ce qui paraîtrait être une "exception culturelle française» : tout d'abord, le cinéma américain est également producteur de comédies à succès, et ce depuis ses débuts, à côté de genres qui ont fait ou continuent à faire la fortune des studios hollywoodiens comme le film d'action, le western ou le film de science-fiction. Ensuite, l'attention portée au cinéma comique français est parfois influencée par une approche critique spécifique: ainsi Philip Powrie (p. I47-I49) remarque-t-il à juste titre que le film de Coline Serreau, Trois Hommes et un couffin (1985), a fait l'objet de nombreuses réflexions critiques en Angleterre et aux États-Unis non seulement à cause de son succès commercial mais aussi parce qu'il se prête tout particulièrement à une approche féministe et qu'il comporte des traits proches de la comédie américaine. Le cinéma comique américain est soumis aux mêmes effets, puisque l'évolution des développements théoriques a contribué à en redéfinir la taxinomie selon Jean-Loup Bourget (p. 17):

[...] le développement des études féministes et de façon plus générale des gender studies [...] tend tout à la fois à reléguer l'aspect social au second plan, à gommer la distinction traditionnelle (d'ailleurs peu nette) entre comédie sophistiquée et comédie loufoque, et à dégager les traits saillants du genre de la comédie romantique (ou romanesque), un genre à vrai dire largement antérieur non seulement aux années 1930, mais à Hollywood.

Il nous a semblé pertinent de concentrer l'analyse sur le corps, un élément essentiel de la comédie qui se situe au croisement de ces perspectives, et d'en dégager les principaux enjeux: le corps comme objet artistique, source de spectacle, marqueur social, ou encore moyen de communication et de cohésion interne à une communauté. Certes, le cinéma ne se distingue pas des autres arts dans son approche inventive et diversifiée de la représentation du corps humain qui s'y trouve surdimensionné comme en sculpture, morcelé comme en peinture, mis en mouvement comme dans la danse, mis en scène comme au théâtre et mis en récit comme en littérature et dans la bande dessinée. Mais une des spécificités du langage cinématographique est, par le recours à l'image photographique, d'être composé, entre autres, de signes indiciels, c'est-à-dire de porter la trace d'un corps réel dont il atteste à la fois l'absence actuelle et la présence passée. La comédie joue sur le paradoxe constitué d'une part par la valeur de cette trace sur le plan physique et symbolique et d'autre part par la déformation apportée par la mise en scène et le montage à cette trace. Contrairement à la transformation en icône du corps, féminin notamment, qui consiste à faire passer pour réelle aux yeux des spectateurs la construction d'un corps idéal, la comédie tend à accentuer les écarts apportés par la représentation à la description du réel. Le jeu entre le visible et le vécu qu'elle propose dégage un sens qui est à la fois le produit et le reflet de 
son contexte socio-culturel. Le rire, propre de l'homme, exploite donc le corps humain, considéré a priori comme donnée «naturelle », biologique, lorsqu'il perd précisément son caractère inné, "normal », et devient un artefact. Le cinéma comique, en général, présente de nombreux exemples de cette "dénaturalisation " du corps humain par la transformation en animal, en machine, en personnage chimérique: ce sont les variantes proposées par les récits cinématographiques français et américains sur ce point, qui pourront fournir quelques pistes pour repérer des spécificités liées au contexte social et culturel de leur production.

La «mascarade » que constitue le travestissement de l'homme en animal, a d'abord obéi à des fins religieuses, parfois stratégiques - le cheval de Troie -, et souvent spectaculaires comme l'attestent de nombreux rituels et fêtes dans le monde. Dans les récits archaïques ou la littérature enfantine, le monde animal et imaginaire symbolise et catalyse la découverte de l'univers et de soimême, dans les premières années de l'existence d'un individu ou d'une civilisation. L'anthropomorphisme qui consiste à assurer la projection de sentiments et comportements humains sur des animaux a perduré dans l'histoire des représentations depuis les mythes et les contes de fées jusqu'aux anime japonais, aux séries télévisées américaines - autrefois Le Cheval de Monsieur Ed (I96I-I966), plus récemment $A L F$ (1986-1990) - et aux films de Walt Disney et de ses émules. Au cinéma, elle peut être source de comique comme l'a prouvé le succès du duo composé par Tom Hanks et son chien dans Turner and Hooch (Roger Spottiswoode, I989) ou celui du chat et de l'âne dans Shrek II (Andrew Adamson, Kelly Asbury, 2004). L'effet comique y est fondé sur la familiarité, c'est-à-dire le fait de retrouver les comportements et la voix de l'être humain sous l'apparence animale 6 .

Mais si ce procédé n'est pas systématiquement source de comique, inversement le comique ne repose pas forcément sur la familiarité. Il tend souvent à utiliser l'animal et l'imaginaire pour ôter à l'humanité son caractère distinctif et, par voie de conséquence, évoquer l'absence d'humanité dans l'expérience individuelle et les relations entre les personnages. La transformation de l'être humain en animal, en monstre ou en robot, caractéristique du genre fantastique et de ses sous-genres (le film d'horreur et le film de science-fiction),

6. À cet égard, le film d'Alain Chabat, Didier (1997), à fois valide et subvertit cette règle. Les indices donnés par le récit filmique, image et son, et par l'expérience des spectateurs concourent à projeter l'image familière et reconstituée d'un chien sur l'image apparente et insolite d'un être humain dont la gestuelle et le comportement sont ceux d'un animal, même s'ils restent filtrés par le regard humain. 
est alors une inversion du procédé de familiarisation, puisque c'est l'étrangeté qui produit l'effet escompté : dans

[...] le film d'épouvante [...] les axiomes de base, les prémisses ou les hypothèses, sont par définition incroyables: existence post mortem, réversibilité de la jeunesse, possession psychique, négation de la mort par le sang, etc. L'effet immédiat et automatique de cet irréalisme est de maintenir le spectateur à distance. (Dadoun, p. I63)

Ainsi la terreur et le rire peuvent-ils être produits tous deux à la fois par la négation de la physiologie du corps humain, sur le plan du contenu narratif, par la distanciation des spectateurs, sur le plan de l'énonciation, et par la mise à nu des "aspects les plus terrifiants de la condition humaine" sur le plan idéologique, au point d'ailleurs de provoquer parfois la confusion chez

[...] ces spectateurs ricanants et bruyants qui exploitent, par la dérision et la grossièreté, la non-participation qu'implique le film d'épouvante. (Dadoun, p. 165)

Ce rapprochement est plus notable dans le cinéma américain, producteur de films fantastiques comme de films comiques, que dans le cinéma français où le film fantastique reste marginal. De fait, le cinéma comique américain a de plus en plus recours aux effets spéciaux pour altérer la représentation visuelle du corps humain, à l'instar du cinéma fantastique, coutumier du procédé depuis longtemps. Reprenant à Swift et à Rabelais la transformation d'échelle du corps humain, le cinéma américain a grandi, grossi, rajeuni, vieilli et rapetissé le corps humain à des fins comiques dans The Nutty Professor (Tom Shadyac, I996), Chérie, jai rétréci les gosses (Joe Johnston, 1989) ou Big (Penny Marshall, p. I988). Il a aussi emprunté à la mise en scène de F. W. Murnau et George A. Romero, au jeu de Bela Lugosi et Christopher Lee, la tendance du fantastique à figer et déformer la face humaine :

[...] dans le film d'épouvante [...], seuls sont tolérés le rictus, l'œil gonflé d'horreur, le hurlement bestial -bref, les masques (comme dans la tragédie antique?). (Dadoun, p. I64)

Le statut indiciel du personnage comique américain a ensuite fait face à une compétition inattendue lorsqu'il a dû partager l'écran avec des personnages de dessin animé dans Who Framed Roger Rabbit (Robert Zemeckis, 1988). Puis son visage a été soumis au remodelage effectué par les effets spéciaux comme celui de Jim Carey dans The Mask (Chuck Russell, I994). Enfin, il ne lui est plus resté d'humain que la voix, certes célèbre, d'un Eddie Murphy ou d'une Cameron Diaz pour interpréter les protagonistes de Shrek (Andrew Adamson et Vicky Jenson, 200I).

Sans doute pour les mêmes raisons techniques, financières et artistiques, qui expliquent sa distance à l'égard du fantastique, le cinéma comique français 
a moins recours aux effets spéciaux en dehors toutefois du dessin animé et de l'adaptation des bandes dessinées. Le film de Jean-Marie Poiré, Les Visiteurs (1993), constitue une exception puisque selon Guy Austin (p. 139) il serait «lié à trois autres genres à part la comédie populaire: le cinéma fantastique, le réalisme poétique et le film historique». Il faut cependant remarquer que les éléments irréalistes du film ont essentiellement pour fonction de déclencher l'action au début du film et d'en clôturer le récit; de plus, ils s'apparentent davantage au merveilleux qu'au fantastique. De fait, dès la naissance du septième art en France, les films de Méliès ont séduit le public en montrant des corps démultipliés, désarticulés, déformés, de Dédoublement cabalistique (1898) à L'Homme à la tête en caoutchouc (I9OI) en passant par L'Homme-orchestre (I900) pour ne citer que quelques titres dans une production prolixe en la matière. Il s'agissait de concurrencer les illusions du théâtre de magie 7 mais aussi les attractions foraines où le corps anormal et non animal des "monstres" était exploité à des fins spectaculaires (la «femme à barbe», «l'homme-éléphant», les jumeaux siamois). Ces derniers, relevant d'une réalité dénaturée, déclenchent l'effroi et s'apparentent ainsi aux personnages fantastiques, tandis que les formes créées par l'illusion et l'artifice déclenchent le rire et caractérisent le film comique dont Max Linder a vite su exploiter la dimension réflexive:

[...] dans ce Max veut grandir qu'on peut par ailleurs " lire " comme une étonnante vengeance du comique sur sa petite taille - voir, à travers elle, sur son statut même de comique -, les efforts du héros pour grandir sont autant d'hommages à différents trompe-l'œil cinématographiques: image allongée, écrasée (quand la tentative échoue) et finalement aussi le simple découpage, dans les scènes, où, pour le croire grandi, on ne voit jamais Max qu'à moitié (dans un plan rapproché). (Král, p. 78)

D'un côté la monstruosité envahit et phagocyte l'humain, de l'autre l'humain se transforme en un soi idéal ou rêvé. D’une certaine façon, le fantastique est du côté de la symbiose et de la contamination, c'est-à-dire de la pathologie et en dernière instance de la destruction de toute humanité, alors que le comique est du côté de la métamorphose et de la substitution, c'est-à-dire du ludique qui suppose le retour au réel.

À ce titre, réduire le corps humain à un type, à une machine, à un objet et, pire encore, à un personnage ${ }^{8}$ participe de la construction comique, comme ont

7. Au théâtre, en revanche, l'intégrité du corps de l'acteur sur scène est garanti : il peut être grimé, déformé, voire désarticulé, il n’en reste pas moins présent et entier comme élément fondamental du spectacle théâtral.

8. Voir P. Sorlin, "A Breath of Sea Air, Jacques Tati's Les Vacances de Monsieur Hulot», French Film: Texts and Contexts, S. Hayward et G. Vincendeau éd., Londres et New York, Routledge, 2000, rééd. 2002, p. IOO-III, sur la réduction de Monsieur Hulot à un personnage 
su l'exploiter Charlie Chaplin dans Les Temps modernes (1936) et Jacques Tati dans ses films. Comme le note Vincent Amiel (p. I5) à propos de Buster Keaton,

[...] la prise en charge par le corps de cette précision et de cette adaptation par ailleurs confiée à la machine est un déni du modèle technologique. Il ne s'agit pas de représenter le corps à l'aune de la machine (comme on pourrait le deviner chez un Vertov au même moment), mais bien de dénigrer implicitement la fonctionnalité de cette dernière grâce à la très précise nécessité interne du genre humain. [...] Ce n'est pas la "machine humaine » qui est l'œuvre ici, c'est la précision de l'élan, la lucidité de la pulsion, l'extraordinaire puissance de perception que la liberté pleinement vécue octroie parfois.

Un des indices qui révèlent la clôture inévitable du jeu comique par opposition à l'entropie fantastique est le traitement de la voix: la voix d'outretombe, la voix du démon, la voix du double caractérisent le film d'épouvante alors que les déformations du corps comique épargnent le plus souvent la voix humaine. Certes les films de Tati paraissent plus radicaux à cet égard, comme le constate André Bazin (p. 46-47),

[...] de la combinaison de ce réalisme et de ces déformations naît l'irréfutable inanité sonore de ce monde cependant humain. Jamais sans doute l'aspect physique de la parole, son anatomie, n’avait été mise aussi impitoyablement en évidence. Habitués que nous sommes à lui prêter un sens même quand elle n’en a aucun $[\ldots]$

nous nous trouvons confrontés à une parole privée de sens, de langage, d'humanité. Mais là aussi, il ne s'agit pas de l'intrusion de l'autre, mais d'un plaidoyer pour que la dénaturation ne soit que temporaire et cède le pas à la véritable humanité. «Le gag de Tati n'est jamais venu de l'idée de savoir comment faire du cinéma drôle mais comment vivre ensemble 9 ", tout comme les parodies de comédie musicale visent à dénoncer la transparence du cinéma hollywoodien pour revenir à la réalité masquée par l'illusion. Marc Cerisuelo (p. 200) montre comment, contrairement à la séquence de doublage de Singinin the Rain (Stanley Donen et Gene Kelly, 1952), la comédie réalisée en 196ı par Jerry Lewis,

The Errand Boy, propose un travail de "défiguration vocale ": l'artifice, pris à sa source filmique (le moment de l'enregistrement), est dénoncé avec une férocité qui n’a d'égale que sa gratuité. Dans un cas l'opération du doublage, enfin démasquée, rendait justice au talent véritable; dans l'autre, l'accent est porté sur le secret de cette activité honteuse que l'audace du cinéaste dévoile en un premier

doté d'un nom, mais privé de la parole, de point de vue et partiellement "privé de personnalité fictionnelle».

9. J.-L. Schefer, «Monsieur Tati», Cahiers du cinéma, nº 342, décembre 1982, p. 32, cité par M. Cerisuelo (p. 174). 
temps (l'on assiste en effet à un véritable doublage qui sauve une interprétation pitoyable) pour mieux la massacrer ensuite.

Mais le rapprochement au niveau des procédés entre films comiques américains et français rend plus marquante encore la différence essentielle en matière d'objectif: de manière très simplificatrice, on pourrait avancer que la comédie américaine vise à l'intégration, alors que la comédie française tend plutôt à renforcer le statu quo ante par le moyen, entre autres, de la dénaturation provisoire. Cette distinction a d'abord un impact sur le plan narratif: la comédie américaine cherche à donner une justification logique au dénouement, tandis que de nombreuses comédies cinématographiques françaises empruntent au théâtre le procédé du deus ex machina pour clore la narration. Les artifices auxquels ont recours les films comiques français font alors souvent preuve d'une imagination débridée, qu'il s'agisse des effets du gène de la malchance dans La Chèvre (Francis Veber, 198I), du partage inconditionnel de la fibre paternelle dans Métisse (Mathieu Kassovitz, 1993), du voyage dans le passé opéré par l'alchimie dans Les Visiteurs, ou de l'apparition d'une Vierge au corps translucide dans Chouchou (Merzak Allouache, 2002).

On notera que, dans ces exemples, la dénaturation du corps humain est utilisée pour constituer la structure même du récit filmique et non pas seulement la caractérisation et l'apparence des personnages: la distance prise avec les données du réel permet d'introduire un dénouement non plus grâce à l'arrivée d'un être divin tombé des cintres, mais par le biais d'une entorse sérieuse aux lois de la physique. Ainsi la malchance devient une maladie contagieuse, un enfant naît de deux pères, les personnages passent du Moyen Âge à l'époque contemporaine et inversement, la Vierge apparaît au protagoniste, musulman de surcroît. S'il s'agit bien évidemment de la reprise par le récit comique de l'opposition classique entre nature et culture, on notera que, pas plus que dans le genre fantastique, la dénaturation du corps humain dans le film comique français ne peut être aussi radicale que dans le film comique américain. Malgré son caractère dérangeant et insolite, elle concourt en fait à illustrer a contrario la pérennité et la stabilité par l'exemple de la tare, de la reproduction, de l'hérédité et de l'anatomie humaine. Ainsi ces films répondent-ils à la fonction sociale de la comédie, selon l'analyse bakhtinienne, en opérant une inversion des rôles qui a pour objectif ultime de renforcer les structures et les rapports de pouvoir d'une société donnée. La faible mobilité des institutions et des classes sociales françaises est en quelque sorte justifiée par l'immuabilité du corps humain dans la comédie, quelle que soit la visée satirique de celle-ci, notamment le refus des différences de race, de genre, de classe. 
Ainsi le film comique français concourt-il finalement à l'acceptation du social au même titre que du naturel par opposition au comique américain voué à promouvoir l'évolution des corps sur l'écran et celle des individus dans le monde référentiel. La différence que peut apporter l'individu au groupe, extravagance tolérée dans la société britannique et rejetée dans la société française, est un facteur de dynamisme essentiel à la société américaine: le rire ne sert pas à isoler mais à reconnaître et valoriser l'étincelle d'humanité qui reste indestructible dans le corps le plus déshumanisé. Une démarche, une voix, un regard survivent toujours au délire, visuel aujourd'hui, narratif autrefois.

Ce qui séduit d'abord, dans la comédie américaine, c'est l'accord d'un style avec un programme, c'est la libération des fantasmes et des comportements qui va de pair avec la dénonciation des hypocrisies, l'effondrement (temporaire sans doute) des clivages de classe, d'âge, de sexe : ce sont les scènes jubilatoires et enfantines de folie collective - ou de folie à deux - qu'on admire chez Capra (Mr. Deeds goes to town, You can't take it with you) comme chez Tay Garnett (Joy of Living), dans Sylvia Scarlett de Cukor comme chez La Cava (Laugh and Get Rich, She Married Her Boss, Fifth Avenue Girl)»(Bourget p. 20).

Le jeu sur la visibilité, source de comique au cinéma, est également un indice intéressant de la conception du corps dans la société et de son utilisation dans le film comique. Le corps nu féminin, offert au regard et donné en spectacle, n'est pas en soi source de comique, mais le devient avec la mise en scène du regard voyeur lorsque celui-ci est involontaire comme lors de la rencontre inopinée d'Harry Sanborn et Erica Barry dans Something's Gotta Give (Nancy Meyers, 2003) ou lorsqu'il est caricaturé dans ses effets comme le portrait de Thérèse peint par Pierre dans Le Père Noël est une ordure (Jean-Marie Poiré, 1982). Surprise et fantasme soulignent l'interdit associé au regard des personnages sur la nudité et le plaisir suscité par sa transgression chez le spectateur. On notera le traitement particulier apporté à la nudité masculine par le film comique français, puisqu'elle subvertit un double tabou dans les sociétés patriarcales occidentales, l'un social, l'autre esthétique. La comédie le met en scène en le désignant et en le transgressant, en révélant et en cachant tout à la fois le sexe masculin par des algues, un short ou un string minuscule dans Les Bronzés (Patrice Leconte et l'équipe du Splendid, 1978), La Cage aux folles (Édouard Molinaro, 1978) ou Tenue de soirée (Bertrand Blier, 1986).

Inversement, la comédie française utilise moins le costume comme marqueur social et source de comique que la comédie américaine, d'une part, parce que le comique français est très souvent verbal ${ }^{\mathrm{Io}}$ et, d'autre part, parce

IO. «Ce qui fait rire les Français est fondé sur de grands écarts linguistiques qui ne fonctionnent que dans leur langue. Une grande part de l'humour français repose sur des jeux de mots intraduisibles" (The things that make the French laugh involve linguistic somersaults that only 
que la fonction d'intégration sociale du comique américain l'incite souvent à recourir au changement, à la mascarade ou à la transformation. Il y a certes quelques exceptions notables du côté français: les films de l'équipe du Splendid et d'Éric Chatiliez -notamment La Vie est un long fleuve tranquille (1988), Tatie Danielle (1990), Tanguy (200I) - ont présenté des personnages affublés de gilets, de salopettes, de blousons noirs, de survêtements et de couvre-chefs pour dénoter leur origine modeste ou arborant collier de perles et costumes trois pièces pour illustrer leur appartenance à la bourgeoisie conservatrice. Dans les films américains, l'habit fait plus souvent le moine et le comique naît, de manière classique, du décalage entre le statut du personnage et sa situation ou son comportement: le travestissement à des fins carnavalesques consiste à habiller le petit escroc en costume sévère et le yuppie prospère en proxénète, puis en clochard dans Un fauteuil pour deux (John Landis, 1983) ou encore de donner à l'étudiante en droit de Harvard l'allure d'une Valley GirlII dans La Revanche d'une blonde (Robert Luketic, 200I). Mais, dans le film français, l'habit est, tout comme le corps, marqué d'une rigidité incontournable, puisqu'il définit le personnage, depuis les vêtements butch de Marijo dans Gazon maudit (Josiane Balasko, 1995), les accoutrements extravagants de Zaza dans La Cage aux folles (ses tentatives de s'habiller en mère respectable échouent l'une après l'autre), le costume de majorette de Frède dans Nuit d'ivresse (Bernard Nauer, 1986), le complet de François Pignon dans Le Dîner de cons (Francis Veber, 1998), et même, d'une certaine façon, les tenues médiévales de Montmirail et Jacquouille dans Les Visiteurs.

Le cas du Père Noël, illustré du côté américain par une séquence d'Un fauteuil pour deux et du côté français par Le Père Noël est une ordure, combine le recours à un costume a priori non marqué socialement et l'utilisation d'un personnage chimérique, plus proche du merveilleux que du fantastique et donc du comique. La mascarade, dans ces deux cas à l'instar de nombreuses autres occurrences dans le cinéma, tient au fait que le costume du Père Noël ou de Santa Claus sert précisément à évacuer tout merveilleux: le Père Noël est devenu une valeur marchande, un accessoire saisonnier dans le cadre de semaines commerciales - le déguisement sert ainsi à rapporter un petit revenu à un individu démuni et sans qualification. Le comique naît de l'incompatibilité entre les connotations du costume - la fête, l'enfance, les cadeaux - et le

work in their own language. Much of French humour is jeux des (sic!) mots, intranslatable worldplay) : "Very droll. The French have jokes, but do they have a sense of humor?», The Economist, 20 décembre 2003, p. 75-76.

II. Cette expression désignait originellement les jeunes filles relativement aisées et oisives de San Fernando Valley, une banlieue résidentielle au nord-est de Los Angeles où l'activité essentielle consistait à faire du shopping dans les grands centres commerciaux. 
personnage qui l'endosse, un SDF violent et sans scrupules dans le film français, un ancien yuppie involontairement réduit à la mendicité dans le film américain. La différence est encore une fois due au dynamisme inhérent à la comédie américaine: le golden boy n'endosse l'habit du Père Noël que pour mieux montrer la progression de sa déchéance. Il commence par dérober la nourriture d'un buffet, puis entre par effraction dans un bureau et finit par tenir toute une foule sous la menace d'une arme à feu, avant que ne prenne place sa rédemption, progressive elle aussi. Dans le film de Poiré, Félix conservera son costume, peu à peu réduit en lambeaux, tout au long du film et son comportement est d'une stabilité remarquable dans l'accumulation des crimes et délits: violences conjugales, meurtre, coups et blessures. On opposera le personnage du Père Noël ainsi redéfini au corps de l'ascensoriste qui, aussitôt entrevu, est privé de vie, dissimulé, découpé et jeté en pâture aux animaux du zoo. Ici la distinction entre l'animal et l'être humain est radicalisée pour produire le comique par l'inversion sans risquer l'ambiguïté. Le comique français est donc fondé sur la logique interne du personnage et du récit - d'où la nécessité d'un deux ex machina pour apporter éventuellement une conclusion ${ }^{\mathrm{I2}}$ : l'immuabilité du corps, du costume et du comportement renvoie à l'idée réconfortante selon laquelle le monde est décodé, familier et inaltérable, dans une société réticente à accepter l'imprévu, l'original, l'absurde³.

Enfin, s’il est un exemple significatif du traitement du corps dans le cinéma comique qui se situe au carrefour de l'opposition entre donnée génétique et apprentissage social, du lien entre pratique individuelle et dynamique sociale, de la différence entre comédie française et comédie américaine, ce pourrait bien être la question de l'orientation sexuelle. Le film noir américain a conféré une fonction narrative normative à quelques exemples de personnages homosexuels :

[...] au niveau de la structure du récit, le personnage homosexuel, même si ce n'est pas lui le méchant (comme le sont Cairo, Bruno, Waldo), incarnera souvent l'une de ces impasses dans le labyrinthe qui retardent la solution du mystère ou mettent en péril la formation du couple hétérosexuel. (Dyer, p. 212)

De même, dans la comédie américaine, le jeu sur l'orientation sexuelle a d'abord pour fonction de redonner une place à ceux qui ne se conforment pas

I2. Cette conclusion est d'ailleurs si peu nécessaire et si artificielle qu'elle permet de donner facilement une suite aux plus grands succès comiques (Les Visiteurs, La Cage aux folles, Les Bronzés).

13. De fait, ce sont des dramaturges étrangers comme Beckett et Ionesco qui ont su faire rire de l'absurde traité par ailleurs sur un registre sérieux par les philosophes existentialistes. 
à l'ordre patriarcal, tandis que, dans la comédie française, c'est le défi à l'ordre biologique qui est accentué et en dernière instance condamné.

Les comédies américaines à très grand succès commercial comme Mrs. Doubtfire (Chris Columbus, 1993) et Tootsie (Sydney Pollack, 1982) ne présentent pas à proprement parler de personnages homosexuels, mais ont recours au travestissement pour proposer une nouvelle perspective au regard des personnages, et donc des spectateurs, sur la différentiation sexuelle et ses effets dans le contexte socio-professionnel. Le corps des personnages reste intact sous le travestissement mais devient un espace d'inscription, avec un accent particulier mis sur la familiarité de la voix comme pour les autres personnages déshumanisés ou dénaturés:

[...] à certaines occasions dans Mrs. Doubtfire et dans d'autres films de «travestis", les voix mâles des "vrais hommes" sous l'accoutrement féminin semblent faire irruption ${ }^{14}$. (Matthews, p. 89)

Comme on l'a vu, la mascarade laisse toujours supposer que le spectacle prendra fin et conduira au retour à la normale, mais aussi à une réévaluation de la norme: il ne s'agit pas véritablement d'explorer la condition des homosexuels ou transsexuels ${ }^{\mathrm{IS}}$, mais la condition faite aux femmes dans une société dominée par l'idéologie patriarcale. D’une certaine façon, l'interchangeabilité des sexes devient dans ces comédies un moyen de soustraire les corps à leurs données biologiques pour formuler une solution alternative aux stéréotypes culturels fondés sur ces données.

Le cinéma comique français contemporain, en revanche, propose davantage de protagonistes homosexuels et semble ainsi vouloir rompre avec la tradition du travestissement qui abonde dans les contes et les comédies de la Renaissance et où «le passage du féminin au masculin est infiniment mieux accepté que son contraire » : l'inversion des rôles, pratiquée dans Trois Hommes et un couffin permet d'éviter le ridicule et la satire et La Cage aux folles atteste du passage du "triomphe d'angoisse» en "fantaisie de triomphe» (Emelina, p. I62). Laccent n'est donc plus mis sur l'«inversion» ludique, mais sur le facteur biologique de l'homosexualité qui y est décrite comme une orientation naturelle à intégrer à la structure sociale majoritairement hétérosexuelle. Dans la comédie douce-amère d'Alain Berliner, Ma vie en rose (1997), la critique des conventions sociales et sexuelles ne remet pas en cause ou n'explicite pas le

I4. On occasions in Mrs. Doubtfire, and in other "cross-dressing" films, the male voices of the "real men" underlying the female apparel seem to burst out.

I5. La mise en scène de protagonistes homosexuels et de leur environnement dans un registre comique est beaucoup plus présente actuellement aux États-Unis à la télévision avec des productions telles que Will and Grace, Queer as Folk, The L Word qu'au cinéma. 
refus de Ludovic d'assumer sa condition biologique. Gazon mandit constitue assurément une exception en accentuant la "fluidité» des déterminations sexuelles ${ }^{16}$, mais en fin de compte Loli reste le personnage emblématique de la femme amante et mère, et la rivalité entre Marijo et Laurent souligne l'alignement des deux personnages sur les stéréotypes masculins, en dépit du retournement final. La Cage aux folles soumet le déroulement du récit et les actions des protagonistes aux enjeux d'un couple hétérosexuel classique, à savoir les responsabilités associées à la paternité. Dans Tenue de soirée, l'homosexualité d'Antoine n'est présentée que comme la substitution d'un partenaire masculin à un partenaire féminin pour Bob et l'expose donc au même traitement: séduction, violence, prostitution. Dans Pédale douce de Gabriel Aghion (I996), la mise en scène de personnages homosexuels a certes pour objectif de remettre en question les préjugés, mais reste subordonnée à l'aventure personnelle des personnages hétérosexuels, Eva, Alexandre et Marie. Quant à Chouchou, les mésaventures d'un jeune homosexuel maghrébin fraîchement arrivé à Paris ne sont imputables, ni à son orientation sexuelle, ni à sa vie de travesti, ni à son statut d'immigré clandestin, mais à l'acharnement d'un fonctionnaire de police atteint de tendances psychotiques contre une psychanalyste. En évitant d'évoquer les problèmes de fond et en jouant sur les stéréotypes sans remettre fondamentalement en cause les préjugés ou la norme, le film comique français pose l'intégration comme un a priori. Sans nier l'affirmation générale selon laquelle,

[...] les comédies filmiques les plus efficaces - tout comme les plus susceptibles de faire réfléchir - sont mimétiques plutôt que didactiques, descriptives plutôt que prescriptives. (Mast, p. 23) ${ }^{17}$

Il apparaît cependant que les choix narratifs effectués par les films français tendent à montrer l'homosexualité comme un fait accepté par la société, alors que les films américains mentionnés tendent à présenter ce consensus comme un objectif, voire un processus en cours et non définitivement acquis tant que la différence entre les sexes reste source d'inégalité et d'injustice.

Les mêmes processus sont à l'œuvre dans les comédies mettant en scène des personnages de races différentes. La comédie américaine tend à souligner la spécificité de personnages afro-américains dans le but de proposer une perspective d'intégration; à cet égard, il est notable que la dimension comique de

I6. Voir D. Waldron, «Fluidity of Gender and Sexuality in Gazon Maudit», France on Film. Reflections on Popular French Cinema, L. Mazdon éd., London, Wallflower Press, 200I, p. 6580.

17. The most effective film comedies - as well as the most thought-provoking ones-are mimetic rather than didactic, descriptive rather than prescriptive. 
films tels que Beverly Hills Cop (Martin Brest, 1984) ou Un fauteuil pour deux repose sur la description de traits particuliers à la communauté noire (lexique, musique, vêtements, voire comportement délinquant) dont la différence tout comme le travestissement et l'homosexualité - sert de prétexte à la valorisation de la norme, c'est-à-dire une société sans préjugés mais aussi sans marginalités. Il est d'ailleurs significatif de constater l'évolution au cinéma du personnage afro-américain qui accède désormais au statut de protagoniste, même si c'est le plus souvent en binôme avec un personnage de race blanche, et n'est plus utilisé pour représenter la part animale de l'être humain. Une des étapes de cette évolution a consisté à dénoncer de manière sarcastique les préjugés racistes, par exemple par le biais de l'inversion grotesque et caricaturale d'un Richard Pryor déguisé en poulet (tout comme son partenaire blanc interprété par Gene Wilder) dans Stir Crazy (Sidney Poitier, I980) : il s'agissait alors de ridiculiser le Noir voulant "passer» pour un Blanc. Men In Black (Barry Sonnenfeld, 1997) est représentatif de plusieurs tendances observables actuellement dans le cinéma commercial américain à cet égard. D'une part, ainsi que l'a démontré Rick Altman ${ }^{18}$, les limites posées par une classification trop rigide continuent à être subverties par la production de films qui, comme $\mathrm{Men}$ In Black, combinent les caractéristiques de plusieurs genres: film d'action, film de science-fiction et film comique. D'autre part, le rôle confié à Will Smith consacre la présence incontestée d'un acteur afro-américain en tête d'affiche qu'il doit cependant partager avec un partenaire blanc, Tommy Lee Jones, contrairement au personnage d'Axel Foley interprété par Eddie Murphy dans Beverly Hills Cop. Enfin, la normalisation du personnage noir, intégré à la mission de défense de la Nation en dépit et à cause de ses spécificités personnelles et culturelles, est accentuée par la présence de marginaux au comportement humain et au corps chimérique. Ces extra-terrestres parfois dangereux parfois facétieux se situent dans la tradition de la comédie américaine évoquée précédemment et contribuent à leur tour au nivellement entre personnages humains et personnages chimériques dans le but d'oblitérer les différences liées à l'apparence physique et à l'origine géographique et culturelle au profit de la reconnaissance de valeurs universelles.

Dans le cinéma comique français, le portrait des personnages d'origine africaine dans Métisse ou Les Keufs (Josiane Balasko, 1987) met au contraire l'accent sur le fait que leur comportement ne differe pas a priori des autres, en dépit de leur apparence physique: l'impact social d'une origine différente n'affecte ni Jamal tout aussi à l'aise dans l'appartement luxueux de ses parents que derrière le comptoir d'un restaurant MacDonald's, ni Lola l'Antillaise

I8. R. Altman, Film/Genre, Londres, BFI, 1999. 
sans souci financier à en juger par son cadre de vie et sa facilité à voyager. Le regard raciste ou sexiste sur les deux inspecteurs de police interprétés par Isaach de Bankolé et Josiane Balasko ne sert pas à revendiquer l'intégration déjà effectuée par leur statut professionnel - mais à accentuer la spécificité biologique des personnages - race ou sexe - dans un contexte donné. Le récit, parce qu'il ne vise pas à représenter un processus, n'a pas besoin de recourir au merveilleux, puisque celui-ci est plutôt utilisé ailleurs pour raconter l'initiation d'un héros dans un monde promis à l'idéal : le recours à l'imaginaire suppose le possible, alors que le réalisme assuré par l'image photographique et les caractéristiques narratives permet de présenter la caractérisation des personnages comme une donnée incontestable du réel. En d'autres termes, par la déformation et la mascarade, la comédie américaine tend à rendre le corps spectaculaire, évident, excessif, donc modifiable, alors que la comédie française tend à l'inscrire comme donnée incontournable et immuable, donc transparente.

On pourrait en déduire que le film comique participe à une définition du multiculturalisme dans chaque société qui se distingue des conceptions traditionnelles: le melting-pot américain garantit les spécificités individuelles dans la mesure où elles sont dotées de dynamisme et s'inscrivent dans la participation de l'individu à l'accomplissement des valeurs définies par la société qui, à son tour, s'enrichit de contributions particulières; en vertu des principes établis par les républiques successives, le multiculturalisme français repose sur un postulat de transparence - l'égalité de tous sans considération de classe, de race, de sexe -, c'est-à-dire sur la séparation absolue entre le civil et le biologique, mais aussi sur le caractère inaliénable et inaltérable de la constitution physique et de la Constitution politique.

Un point essentiel reste néanmoins à développer pour répondre à la question du particularisme culturel dans le cinéma comique: si on distingue des différences fondamentales au niveau de la composition narrative de ces films, il est tout aussi évident que les spectateurs sont amenés à dépasser ces particularités et ces différences comme l'atteste le succès des comédies américaines et de leurs stars, Whoopi Goldberg, Robin Williams, Cameron Diaz et autres Adam Sandler. Certes, l'inverse n'est pas toujours vrai, puisque les remakes de films français comme Le Père Noël est une ordure, Trois Hommes et un couffin et Les Visiteurs n'ont pas séduit les spectateurs d'outre-Atlantique à la mesure de leurs versions originales; quant aux grands favoris de la critique et du public français, Jerry Lewis et Woody Allen, ils sont loin de bénéficier de la même reconnaissance dans leur propre pays. Pourtant s'il est un produit distribué à l'échelle globale aujourd'hui, c'est bien le cinéma américain en 
général et les comédies qu'il propose en particulier: doit-on alors y repérer la présence de traits communs à une humanité - le rire et le cinéma comme sources de plaisir et de cohésion sociale?

\section{BIBLIOGRAPHIE}

Amiel V., Le Corps au cinéma. Keaton, Bresson, Cassavetes, Paris, Presses universitaires de France, 1998.

Austin G., Contemporary French Cinema: an Introduction, Manchester, Manchester University Press, 1996.

Bazin A., «Monsieur Hulot et le temps», Qu'est-ce que le cinéma, Paris, Cerf, rééd. 1994. Bourget J.-L., Hollywood. La norme et la marge, Paris, Nathan, 1998.

Cerisuelo M., Hollywood à l'écran, Essai de poétique historique des films: l'exemple des métafilms américains, Paris, Presses de la Sorbonne nouvelle, 2000.

Dadoun R., Cinéma, psychanalyse et politique, Paris, Séguier, 2000.

Dyer R., «Homosexualité et film noir (Gilda)», Revoir Hollywood. La nouvelle critique anglo-américaine, N. Burch éd., Paris, Nathan, 1993.

Emelina J., Le Comique. Essai d'interprétation générale, Paris, SEDES, I99I.

Hayward S. et G. Vincendeau éd., French Film: Texts and Contexts, Londres et New York, Routledge, 2000, rééd. 2002.

King G., Film Comedy, Londres, Wallflower Press, 2002.

Král P., Les Burlesques ou Parade des somnambules, Paris, Stock, 1986.

Mast G., The Comic Mind: Comedy and the Movies, Chicago, Chicago University Press, 1997.

Matthews N., Comics Politics: Gender in Hollywood Comedy After the New Right, Manchester, Manchester University Press, 2000.

Powrie P., French Cinema in the Ig8os: Nostalgia and the Crisis of Masculinity, Oxford et New-York, Clarendon Press, 1997.

Prédal R., Le Jeune Cinéma français, Paris, Nathan/VUEF, 2002.

Waldron D., "Fluidity of Gender and Sexuality in Gazon Maudit», France on Film. Reflections on Popular French Cinema, L. Mazdon éd., Londres, Wallflower Press, 200I, p. 65-80.

\section{FilMOGRAPHIE}

Beverly Hills Cop (Martin Brest, 1984)

Big (Penny Marshall, 1988)

Les Bronzés (Patrice Leconte et l'équipe du Splendid, 1978)

La Cage aux folles (Edouard Molinaro, 1978)

La Chèvre (Francis Veber, 198I)

Chérie, jai rétréci les gosses (Joe Johnston, 1989)

Chouchou (Merzak Allouache, 2002). 
Dédoublement cabalistique (Georges Méliès, I898)

Didier (Alain Chabat, 1997)

Le Dîner de cons (Francis Veber, 1998)

The Errand Boy (Jerry Lewis, 196I)

Fifth Avenue Girl (Gregory La Cava, 1939)

Gazon maudit (Josiane Balasko, 1995)

L'Homme à la tête en caoutchouc (Georges Méliès, I9OI)

L'Homme-orchestre (Georges Méliès, I900)

Joy of Living (Tay Garnett, 1938)

Les Keufs (Josiane Balasko, 1987)

Laugh and Get Rich (Gregory La Cava, 193I)

The Mask (Chuck Russell, 1994)

Ma vie en rose (Alain Berliner, 1997)

Max veut grandir (Max Linder, I9I2)

Men In Black (Barry Sonnenfeld, 1997)

Métisse (Mathieu Kassovitz, I993)

Mr. Deeds goes to town (Frank Capra, 1936)

Mrs. Doubtfire (Chris Columbus, 1993)

Nuit d'ivresse (Bernard Nauer, 1986)

The Nutty Professor (Tom Shadyac, 1996)

Pédale douce (Gabriel Aghion, 1996)

Le Père Noël est une ordure (Jean-Marie Poiré, 1982)

La Revanche d'une blonde (Robert Luketic, 200I)

She Married Her Boss (Gregory La Cava, 1935)

Shrek (Andrew Adamson et Vicky Jenson, 200I)

Shrek II (Andrew Adamson, Kelly Asbury, 2004)

Singin'in the Rain (Stanley Donen et Gene Kelly, 1952)

Something's Gotta Give (Nancy Meyers, 2003)

Stir Crazy (Sidney Poitier, 1980)

Sylvia Scarlett (George Cukor, 1935)

Tanguy (Eric Chatiliez, 200I)

Tatie Danielle (Eric Chatiliez, I990)

Les Temps modernes (Charlie Chaplin, 1936)

Tenue de soirée (Bertrand Blier, 1986)

Tootsie (Sydney Pollack, I982)

Trois Hommes et un couffin (Coline Serreau, 1985)

Turner and Hooch (Roger Spottiswoode, 1989)

Un fauteuil pour deux (John Landis, 1983)

Les Vacances de Monsieur Hulot (Jacques Tati, I953)

La Vie est un long fleuve tranquille (Eric Chatiliez, I988)

Les Visiteurs (Jean-Marie Poiré, 1993)

Who Framed Roger Rabbit (Robert Zemeckis, 1988)

You can't take it with you (Frank Capra, 1938) 